\title{
Mobile Devices Risks and Recommendation
}

\author{
Ioan ADASCALITEI \\ Bucharest University of Economic Studies \\ ioan.adascalitei@ie.ase.ro,
}

Versatile application security is a measure to make sure about applications from outside dangers like malware and other advanced fakes that hazard basic individual and money related data from programmers. Portable application security has gotten similarly significant in this day and age. A penetrate in versatile security cannot just give programmers access to the client's very own life progressively yet in addition reveal information like their present area, banking data, individual data, and significantly more. In this paper are presented security risks for Android ecosystem, for the iOS ecosystem and also some common risks for both platforms and recommendations in order to avoid this security flaws.

Keywords: Android security risks, iOS security risk, mobile applications, countermeasures DOI: $10.24818 /$ issn14531305/24.3.2020.05

\section{Introduction}

In 2018, portable applications were downloaded onto client gadgets more than 205 billion times. Information by Marketing Land shows that 57 percent of all out computerized media time is spent on cell phones and tablets [1]. As a general rule, our day by day lives rely upon applications for texting, web based banking, business capacities, and versatile record the executives. As per Juniper Research, the quantity of individuals utilizing portable banking applications is moving toward two billionaround 40 percent of the world's grown-up populace.

Engineers give careful consideration to programming configuration so as to give us a smooth and helpful experience. Individuals readily introduce versatile applications and give individual data, however infrequently stop to consider the security suggestions.

Clients take part in about all exercises on cell phones, directly from viewing the news to browsing messages, texting, buying things on the web, and doing bank exchanges. Through these applications, organizations can accumulate usable data, for example, the area, use insights, telephone number, preferences, detests, and other important measurements about clients, which can assist organizations with settling on exact choices to improve their administrations. On the off chance that the information in these cell phones go in an inappropriate hands, it very well may be destructive to the client.

Along these lines, the requirement for portable application security has gotten inescapable.

\section{Android App Security Risks Reverse engineering}

Android applications are created in Java with a coordinated improvement condition (IDE) like Eclipse. These Java applications are switched with different devices accessible on the net. With Android, the bytecode is changed and pressed again as APK documents. Turning around Android applications can without much of a stretch give test login qualifications, bits of information into terrible structure, insights concerning the libraries and classes utilized. It can likewise give insights regarding the kind of encryption utilized within the application. This will help the aggressor is not just hacking one gadget yet various gadgets utilizing an identical decoding technique. 


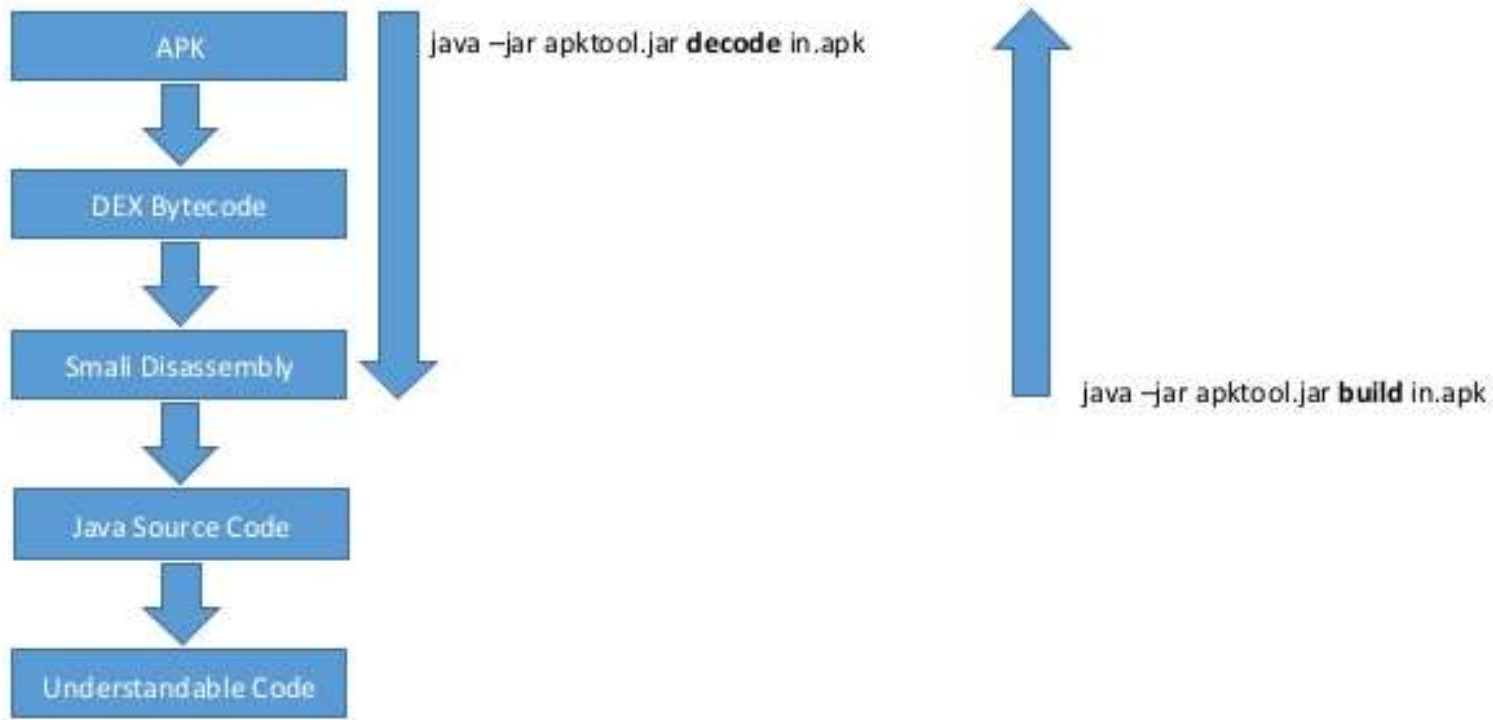

Fig. 1. Reversing an APK [2]

\section{Insecure Platform Usage}

Android OS and applications become helpless against the OWASP Mobile Top 10 dangers when application designers disregard the prescribed procedures distributed by Google to talk with its versatile OS, especially through unbound Android aims and stage authorizations. for example, when the designer doesn't confirm about sent out administrations or issues an off-base banner to an API call, their application stands presented to programmers. Programmers will normally sneak on Android gadgets to urge BroadcastReceiver cases which are intended for authentic applications. Designers will normally disregard the use of LocalBroadcastManager to send and obtain messages for authentic applications, consequently making a security lacuna.

\section{Ignoring Updates}

Numerous Android designers don't refresh their applications consistently or pay notice to the OS patches gave by Android, which brings about an absence of insurance against recently discovered vulnerabilities. Updates spread the foremost recent security fixes and disregarding the equivalent can open applications to the foremost recent security dangers.

\section{Rooted Devices}

The Android OS lets clients root their gadgets utilizing outsider applications with some notice gave to them. Be that because it may, only 1 out of each odd client comprehends that their attached gadget opens it to manage from programmers and malware. For engineers, it, hence, becomes fundamental either to not permit their application to run in a longtime domain or issue ordinary alerts to clients. 


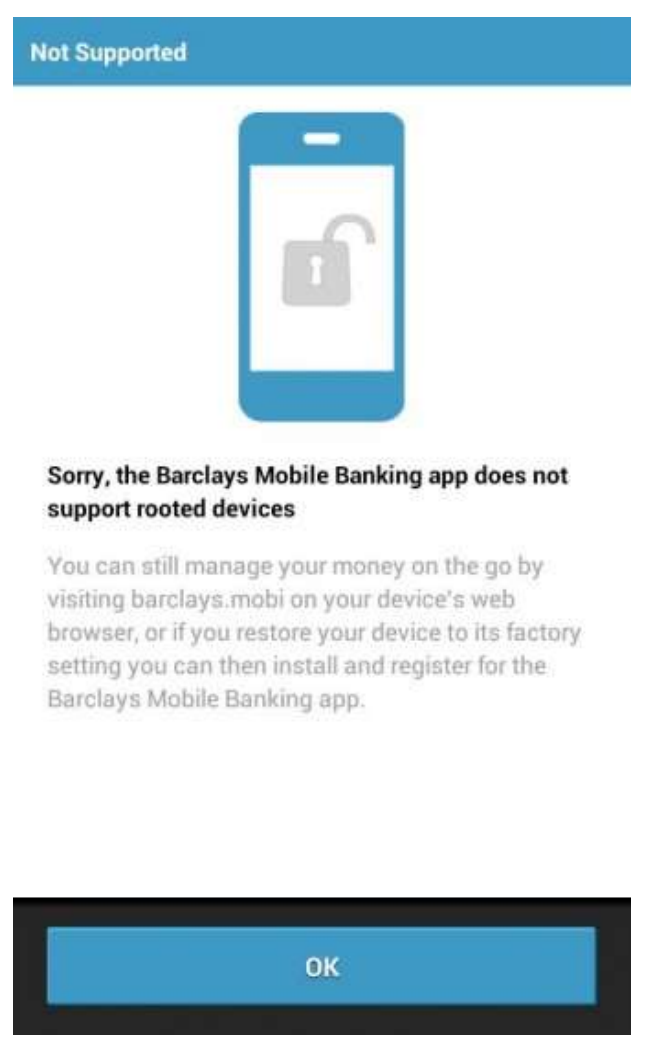

Fig. 2. Rooted device

\section{3 iOS App Security Risks}

In contrast to Android, Apple iOS working framework carefully upholds security includes and could be a shut working framework. Applications can't speak with different applications or straightforwardly get to the indexes or information of various applications. iOS applications are created in local Objective $\mathrm{C}$ language with devices like Xcode. It depends on an analogous ARM rendition of XNU bit as that of OSX, which is employed in Apple's workstations and Mac PCs.

\section{Jailbreak}

Jailbreaking could be a well-known term utilized with regards to Apple gadgets. It includes finding a trial within the part that allows clients to run unsigned code on cell phones. Jailbreaking is fastened, which suggests that every time a client reboots their telephone, it should be related to a $\mathrm{PC}$ or run a jailbroken code. While untethered escape implies that the code will remain the phone significantly after a reboot.

\section{User Authentication}

iOS offers gadget level security through Face ID and Touch ID and cases that they're secure considering the very fact that they utilize a processor break away the rest of the OS. it's called the Secure Enclave, which runs on a committed microkernel. In any case, programmers have indicated that Touch ID are often undermined, most quite with a gadget called GrayKey, which makes beast driving the password speculating simple by getting eliminate the necessity to carry up between endeavors at speculating. When application designers use Touch ID frameworks to make sure information or administrations inside their applications, they're likewise presented to the present quite defenselessness.

\section{Insecure Data Storage}

Most applications store information in SQL databases, treats, paired information stores, or even as basic content. These capacity areas can be gotten to by programmers when the working framework, structure, or compiler is helpless. Additionally, jailbreaking gadgets lead to information presentation. At the point 
when programmers access the database, they change the application and gather the data on their machines. Jailbroken gadgets uncover even the most complex encryption calculations.

Security specialists have likewise discovered that unreliable information stockpiling is one of the most well-known vulnerabilities in iOS gadgets, which programmers endeavor to take passwords, monetary data, and individual information or clients.

\section{Common App Security Risks}

\section{Lack of encryption}

Encryption is a technique for moving information in figured code which can't be seen without coordinating it with a mystery key. As indicated by information by Symantec, about 13.4 percent of customer gadgets and 10.5 percent of big business gadgets don't have encryption empowered, which can without much of a stretch uncover touchy information as plain content. Utilizing an elevated level of information encryption guarantees that the application can't be effortlessly split.

\title{
SCARER S S INFO SECURITY.
}

\section{Weak Encryption Leaves Mobile Health App at Risk for Hacking}

\author{
DHS, Philips Issue Advisories for HealthSuite Android Health App
}

The Android app enables patients to monitor and track their health habits with various connected Philips devices that measure heart rate activity, sleep, blood pressure, weight and body composition analysis.

"Successful exploitation of this vulnerability may allow an attacker with physical access to impact confidentiality and integrity of the product," DHS notes in its alert. "The software uses simple encryption that is not strong enough for the level of protection required."

Fig. 3. Weak Encryption [3]

\section{Malicious code injection}

Client structures can be effectively used to infuse noxious code and access the server information. For instance, certain applications don't limit the characters a client can include in a field. This permits programmers to infuse a line of JavaScript in to the login structure and access private data. 


\title{
threat post
}

\section{WhatsApp Bug Allows Malicious Code- Injection, One-Click RCE}

\author{
Security researchers have identified a JavaScript vulnerability in the WhatsApp desktop \\ platform that could allow cybercriminals to spread malware, phishing or ransomware \\ campaigns through notification messages that appear completely normal to unsuspecting \\ users. And, further investigation shows this could be parlayed into remote code-execution.
}

Fig. 4. Malicious Code-Injection [4]

\section{Binary planting}

It is a general term where an aggressor puts a parallel document containing malevolent code on a neighborhood record framework in the cell phone and afterward executes it to oversee the gadget. This should be possible with the assistance of a pernicious SMS or compelling the client to tap on noxious connections. Along these lines, programmers can put malevolent code even in authentic organizers or inside installer documents and execute it voluntarily, therefore trading off the gadget security. Double planting can prompt figuring out also, where aggressors attempt to deconstruct the code of an application and access the center code. When the code is uncovered, programmers can control it to discover the vulnerabilities and endeavor it for additional malevolent activity.

\section{ZDNet}

\section{Npm team warns of new 'binary planting' bug}

\author{
Npm (Node.js Package Manager) devs say the npm command-line \\ interface (CLI) client is impacted by a security bug - a combination between a file traversal and an \\ arbitrary file (over)write issue.
}

The bug can be exploited by attackers to plant malicious binaries or overwrite files on a user's computer.

Fig. 5. Binary planting [5]

\section{Mobile botnets}

They are a sort of bots that sudden spike in demand for IRC systems made with the assistance of Trojans. At the point when a tainted gadget associates with the web, it begins to fill in as a customer and sends data to a server. Versatile botnets plan to deal with the gadget and can be utilized to send messages and instant messages, make calls, and access individual information, as photographs and contact records. 


\section{ITPro.}

\section{loT botnets are on the rise and $5 \mathrm{G}$ isn't helping anything}

Referencing figures from Nokia's 2019 Threat Intelligence Report. McNamee said the telecoms glant observed $78 \%$ of botnets carried active malware. $35 \%$ of which shared similarities in either code or attack methodology with 2016's Mirai.

Fig. 6. IoT botnets [6]

\section{Recommendations}

The accepted procedures of portable application security guarantee that the application is without chance and doesn't unveil the individual data of the client. It is significant for engineers to guarantee that all security checks are performed before the application is transferred for open utilization. The engineer ought to consider the accompanying techniques to guarantee that their shopper and business applications are not inclined to unapproved access by corrupt components.

\section{Enhance Data Security}

Information security strategy and rules ought to be set up to guarantee clients can without much of a stretch abstain from getting trapped in the snare of programmers. This can incorporate having very much executed information encryption when the data is moved among gadgets and utilizing firewalls and security devices at whatever point important. You can allude to the rules set down for Android [7] and iOS [8].

\section{Not Saving Passwords}

Numerous applications demand clients to spare passwords so as to keep them from over and again entering the login accreditations. In an occasion of versatile robbery, these passwords can be gathered to access individual data. Additionally, if the secret phrase is spared in a decoded design, the odds of them being gathered are high. To keep this from occurring, engineers should abstain from sparing passwords on cell phones. Rather, they ought to be saved money on the application server, with the goal that the influenced clients can transform them by signing on to the server regardless of whether the cell phone is absent.

\section{Enforce Session Logout}

It is frequently observed that clients neglect to $\log$ out of the site or application they are utilizing. On the off chance that it is a banking application or some other installment application, this can be hurtful. Thus, installment applications will in general end the meeting of a client after a specific time of latency or on each logout for expanded security. Designers must uphold a meeting logout on all business and customer driven applications, regardless of whether they anticipate that their clients should be exceptionally educated.

\section{Consult Security Experts}

Regardless of how encountered an inward security group is, an outside perspective on the applications can give an alternate point of view. There are a few security organizations and applications which can be sent in recognizing the escape clauses and diminish the odds of getting traded off. Organizations ought to urge their advancement groups to get the security highlights of their applications evaluated by outsider specialist organizations.

\section{Apply Multi-Factor Authentication}

Multifaceted validation includes an additional layer of security when a client signs into an application. The multifaceted validation strategy additionally conceals for powerless passwords which can be handily speculated by 
programmers and bargain the security of an application. The multifaceted verification gives a mystery code that must be entered alongside the secret phrase to sign into a gadget or application. This code is either sent through SMS, email, Google Authenticator or biometric strategies. Not upholding multifaceted verification on the application can permit programmers to figure powerless passwords.

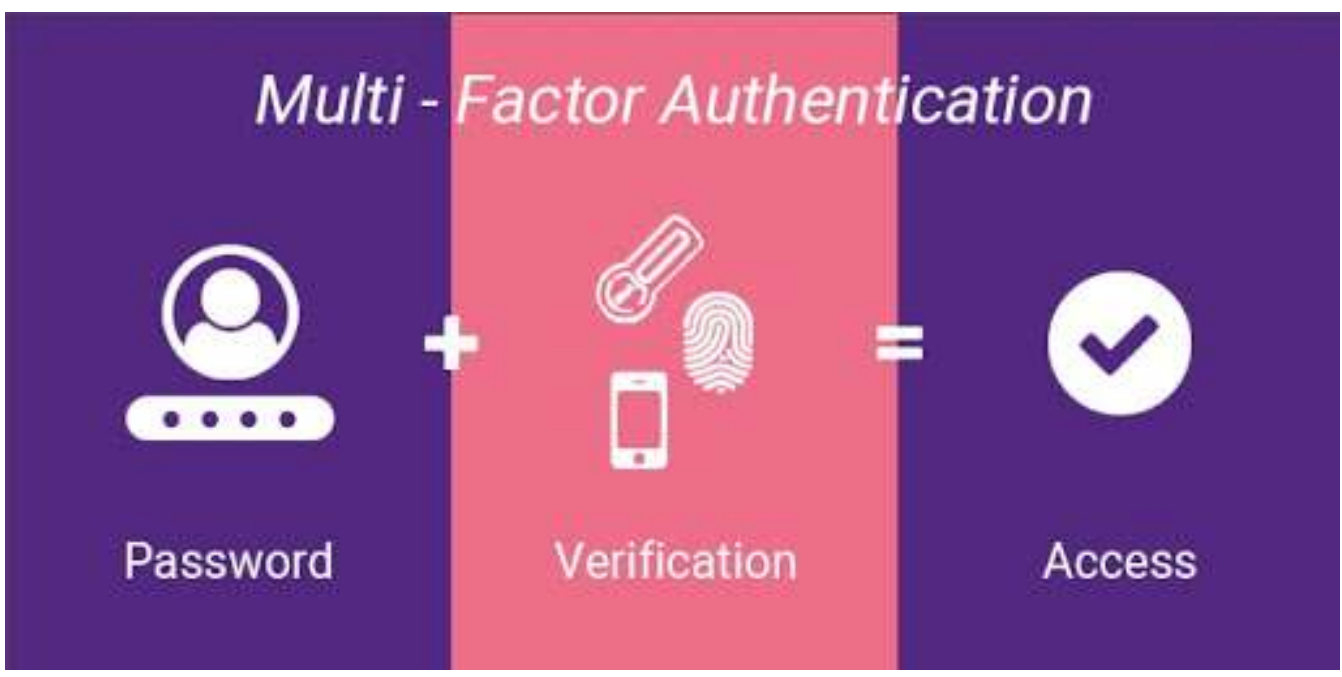

Fig. 7. Multi Factor Authentication [9]

\section{Penetration Testing}

Infiltration testing is done to check known vulnerabilities in an application. It expects to discover potential shortcomings that an aggressor may utilize and bargain the security of the last application. It includes checking frail secret key approach, decoded information, authorizations to outsider applications, no secret key expiry convention, and so on. By reproducing the demonstrations of a potential programmer, the security group decides whether there is any shortcoming in the application. It is suggested that infiltration testing is performed consistently to keep the application secure. White box testing and discovery testing are different kinds of infiltration testing estimates that can be attempted to check for security issues.
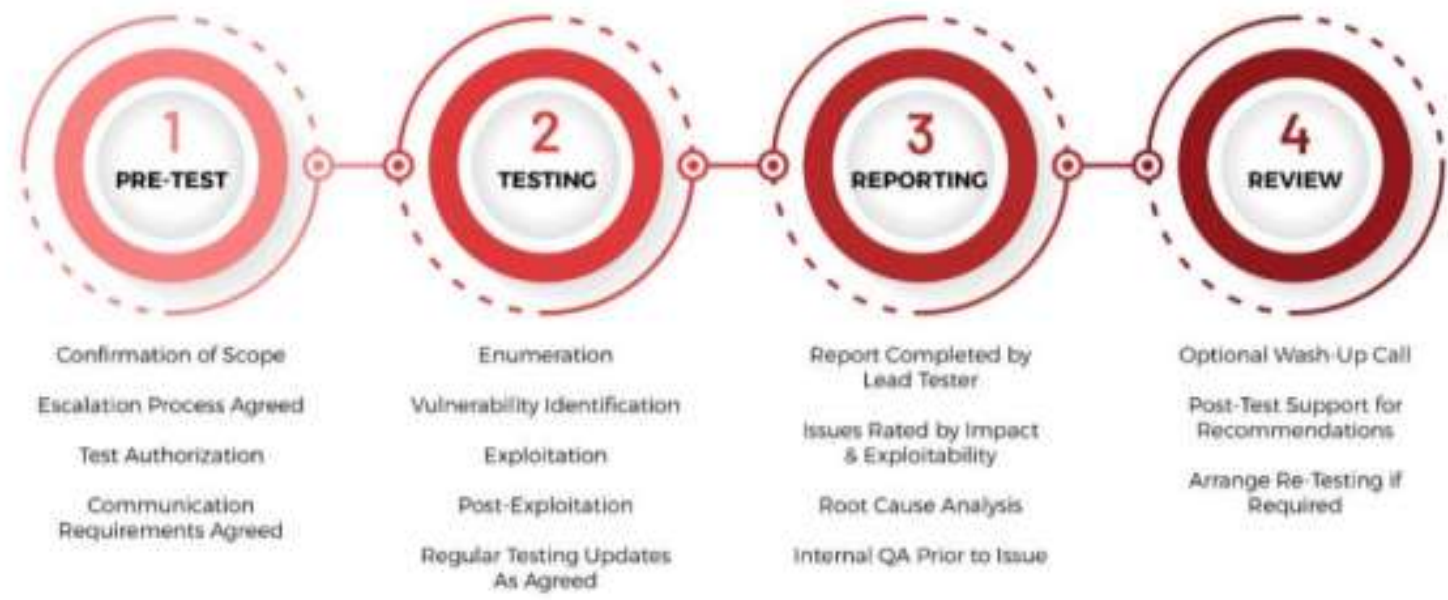

Fig. 8. Penetration Testing [10]

\section{Prevent Usage of Personal Devices}

To forestall the overhead expense of purchasing frameworks, numerous organizations like to request that their representatives bring their own PCs or shrewd gadgets for improvement. This may open the system to a huge amount of contaminations that may have been assembled on a worker's gadget. Malware and Trojans venture out starting with one gadget then onto the next as 
such. Subsequently, it is imperative to have a security approach set up and forestalls such practices. Every gadget associating with an office system ought to be filtered altogether with firewall, antivirus, and hostile to spam programming or ought not to be permitted to interface by any stretch of the imagination.

\section{Utilize Third-Party Libraries with Precaution}

Utilizing outsider libraries may lessen the measure of coding done by the engineer and facilitate the application advancement process. Be that as it may, it very well may be a dangerous suggestion. For instance, the GNU C library had a security defect that permitted cradle flood, which programmers could endeavor to remotely execute a malignant code and crash a gadget. It went on for a long time before the open-source network that adds to the GNU Project discharged a fix in 2016 [11]. Hence, engineers should constrain the utilization of various libraries and make a strategy for taking care of libraries so as to make sure about applications from assaults.

\section{Restrict User Privileges}

The more benefits a client is given the more are the odds of getting the security of an application endangered. On the off chance that the client with a high number of benefits is hacked, programmers can do an unfathomable degree of harm to the application. Essentially, an application ought to likewise not request benefits on a gadget for capacities it doesn't require: for instance, benefits to understand SMS, DCIM envelope, and so forth.

\section{Session Handling}

Meetings on cell phones last any longer in contrast with work areas. This builds the server load. Utilizing tokens rather than gadget identifiers to make a meeting is an increasingly secure alternative. Tokens can be denied at whatever point required and are increasingly secure in the event of a lost or a taken gadget. Designers ought to likewise consider meeting lapse as a choice.
Empowering remote cleaning of information for lost and taken gadgets is likewise a decent wellbeing choice to keep in the application.

\section{Manage Keys Securely}

Key administration is urgent for encryption. Hard coding keys are destructive to the application's security and ought to be evaded by engineers. In the event that somebody takes the key, they can without much of a stretch oversee the gadget. Keys ought to be put away in a protected compartment and for the most part not on the client's gadget. A portion of the famously utilized cryptographic conventions for this reason for existing are MD5 hash and SHA1. Designers should utilize the most recent encryption norms and APIs, for example, 256-piece encryption with SHA-256 hashing.

\section{Test Apps Periodically}

Making sure about a versatile application is anything but a one-time process. New dangers develop every day and updates to fix these dangers are required before they can make any harm the client's gadget. Breaks like the spread of ransomware WannaCry and NotPetya, which scrambled clients' Windows gadgets and requested a payoff in bitcoins, in 2016 and 2017 caused enough caution in the engineer network for them to pay attention to cybersecurity [12]. In spite of the fact that this ransomware to a great extent influenced work areas, the quickness and viability of their spread show the requirement for occasional testing of applications, as new dangers are consistently round the corner.

\section{Ensure HTTPS Communication}

It represents Hypertext Transfer Protocol Secure and is appeared differently in relation to HTTP correspondence. HTTPS offers the security of information when it is transmitted over a system. The correspondence convention is encoded by Transport Layer Security (TLS). TLS and Secure Socket Layer (SSL) are cryptographic conventions that guarantee information protection over different correspondence channels. Then again, HTTP information is decoded, 
unvalidated, and strange, which permits programmers to keep an eye on client content. Designers must guarantee a legitimate SSL declaration on the server to which the application is associated and send information between the application and the server just utilizing the HTTPS convention.

\section{Encrypt Cache}

The reserve is a product segment that spares the information briefly on the client's gadget. This is utilized to forestall the deferral of information recovery. Programmers can without much of a stretch access information put away in store on the off chance that it isn't encoded. On occasion the application doesn't evacuate its information after a meeting closes, and the store doesn't lapse. In the event that these store records get into inappropriate hands, programmers can control it to get to client information or the server.

\section{Code Obfuscation}

Probably the most ideal approaches to shield an application from programmers is to utilize

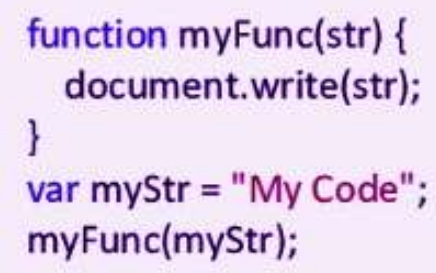

(a) original code code muddling procedures. It is a demonstration of making a code that is hard for programmers to comprehend. This strategy has gotten well known and is utilized to disguise code from assaults. Obfuscators are utilized to consequently change over programming code into an organization that can't be comprehended by people. Code obscurity incorporates:

- Scrambling a few or the whole code

- Expelling metadata which may uncover data about the libraries or APIs utilized

- Renaming classes and factors so they can't be speculated

Code is muddled to keep information and property from programmers who may figure out code utilizing programming programs. In Apple's iOS, this strategy isn't so across the board as its libraries are shut. Then again, Android has open-source libraries. Thus, it is fundamental for Android designers to muddle code.

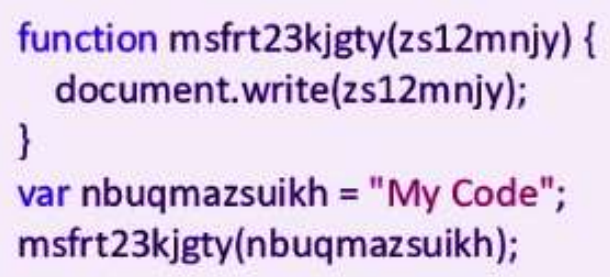

(b) obfuscated code

Fig. 9. -Example of obfuscated code

\section{Conclusion}

Portable application designers ought to naturally realize that as their applications accumulate significance in the gadgets of clients, programmers start to get intrigued too. As depicted above, programmers attempt to abuse vulnerabilities in applications or gadgets utilizing the manual just as computerized apparatuses. Accordingly, it is significant for designers to test their applications altogether before they are transferred to application stores.

At long last, organizations ought to comprehend that the effect of portable application security goes past client security and effects the notoriety of the brand generally speaking. With the expanding hacking endeavors and information penetrates, clients know about versatile application security issues and incline toward applications which are secure over those which can take their data. Subsequently, application designers ought to endeavor to make applications which fulfill the necessities of the client and spotlight their endeavors on the security angle too. 


\section{References}

[1] J. Clement. (2020, January) https://www.statista.com. [Online]. https://www.statista.com/statistics/271644/w orldwide-free-and-paid-mobile-app-storedownloads/

[2] Tom Keetch. (2016, April) https://www.slideshare.net/. [Online]. https://www.slideshare.net/TomKeetch/st eelcon-2015-reverseengineeringobfuscated-android-applications

[3] Marianne Kolbasuk McGee. (2018, December)

https://www.careersinfosecurity.com.

[Online].

https://www.careersinfosecurity.com/wea k-encryption-leaves-mobile-health-appat-risk-for-hacking-a-11833

[4] Elizabeth Montalbano. (2020, February) https://threatpost.com.

[Online]. https://threatpost.com/whatsapp-bugmalicious-code-injection-rce/152578/

[5] Catalin Cimpanu. (2019, December) https://www.zdnet.com. [Online]. https://www.zdnet.com/article/npm-teamwarns-of-new-binary-planting-bug/

[6] Conner Jones. (2019, October) https://www.itpro.co.uk. [Online]. https://www.itpro.co.uk/security/34703/io t-botnets-are-on-the-rise-and-5g-isn-thelping-anything

[7] Google. https://developer.android.com. [Online]. https://developer.android.com/topic/secur ity/best-practices

[8] Apple. https://developer.apple.com. [Online]. https://developer.apple.com/documentati on/security

[9] Nelson Cicchitto. (2018, March) https://www.avatier.com. [Online]. https://www.avatier.com/blog/definingmulti-factor-authentication-need-now/

[10] Robert Bond. (2019, November) https://secureops.com/. [Online]. https://secureops.com/penetrationtesting/va-vs-pt2/

[11] Noah Gamer. (2016, February) https://blog.trendmicro.co. [Online]. https://blog.trendmicro.com/severesecurity-flaw-found-in-linux-library/

[12] Wikipedia. (2020, May) https://en.wikipedia.org. [Online]. https://en.wikipedia.org/wiki/WannaCry_ ransomware_attack

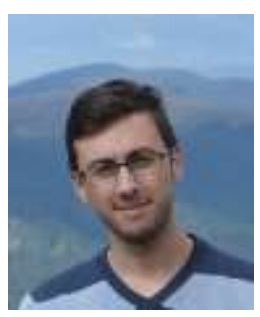

Ioan ADĂSCĂLIȚEI is a PhD Student at the Economic Informatics Doctoral School and his theme is "Security of mobile-based systems". Currently he is Android Programmer at wirtek. He is interested in Mobile Development, including Android and iOS and mobile Security. 